\title{
Making features similar: Comparison processes affect perception
}

\author{
RAN R. HASSIN \\ New York University, New York, New York
}

\begin{abstract}
In this paper, the constructive nature of comparison processes (both similarity- and differenceoriented judgments) is examined through their effects on visual perception. Previous research has shown that comparison processes enhance the tendency to interpret ambiguous objects in the light of the unambiguous objects with which they are compared (Medin, Goldstone, \& Gentner, 1993). In the present paper, it is argued that comparison processes affect not only the interpretation of objects, but also their perception. In addition, it is argued that the perceptual effects of similarity-oriented comparison processes differ from those of difference-oriented comparison processes. Accordingly, it is demonstrated that when estimation of an object's size is preceded by similarity-orientedcomparisons, the Ebbinghaus illusion practically disappears, whereas prior difference-oriented comparisons tend to enhance the illusion.
\end{abstract}

Similarity is a cornerstone of a wide range of psychological theories. Thus, for example, it plays an important role in categorization (Smith \& Medin, 1981), in problem solving (Holyoak \& Koh, 1987), in transfer of learning (Osgood, 1949), in shaping beliefs (Osherson, Smith, Wilkie, Lopez, \& Shafir, 1990), in metaphor understanding (Ortony, 1979), and in transference (Andersen, Glassman, Chen, \& Cole, 1995). Indeed, it seems that "the concept of similarity is ubiquitous in psychological theory" (Tversky, 1977, p. 327).

\section{Similarity and Difference}

The suggestion that similarity and difference judgments are closely interrelated processes seems to be a psychological truism: One cannot note similarities without acknowledging differences, and vice versa ${ }^{1}$. However, although at first sight it might seem that studying one of these processes (e.g., similarity) will suffice to understand and predict both (the similarity and difference are complementary view), current theories and findings make it clear that this is not always the case. For example, Tversky (1977) demonstrated that when the similarity and difference of different pairs are compared, those pairs that are more familiar may be judged as both more similar and more different than those that are less familiar (see also Medin, Goldstone, \& Gentner, 1990). Similarly, Gentner and Markman (1994) showed that differences are easier to find for similar pairs than for dissimilar pairs. Thus, it seems that similarity and differ-

I thank Benny Shanon and the late Amos Tversky for discussions of some of the ideas presented in this paper and Ido Levitan and Sarit Shaanani for their help in preparing and conducting the studies. Correspondence concerning this article should be addressed to R. R. Hassin, Department of Psychology, New York University, 6 Washington Place, 7th Floor, New York, NY 10003 (e-mail: ran.hassin@nyu.edu). ence judgments are not simple inverses of each other: They are processes that interact in complex ways. In light of these intimate relationships, I will henceforth use the term comparison processes to refer to the processes of judging either similarity or difference. In the following terminology, comparison processes can be either similarity oriented or difference oriented.

\section{The Present Research}

In the present study, I examine not how features affect comparison processes, but how comparison processes affect the perception of features. Previous research (Medin, Goldstone, \& Gentner, 1993) has demonstrated that the interpretation of ambiguous stimuli is affected by the properties of the unambiguous stimuli with which they were compared. This tendency was more evident when participants listed similarities between the objects than when they listed differences. The present study takes the examination of the constructive role of comparison processes one step further, by examining their role in the perception of features. It is hypothesized that the effects of comparison processes on perception are similar to those they have on interpretation. Hence, similarity-oriented comparison processes should render the features of the objects in the comparison more similar to each other, relative to their perception in a difference-oriented comparison process.

The perceptual effect of comparison processes is examined here through the Ebbinghaus illusion. The illusion is demonstrated in Figure 1. While examining the two figures, compare the central (target) ellipse in Figure 1A with that in Figure 1B. Despite the fact that these two ellipses are identical in size, the one in Figure 1B looks bigger. This illusion presumably results from a perceptual contrast effect between the target and the context: When the context is composed of relatively large stimuli, the 
A
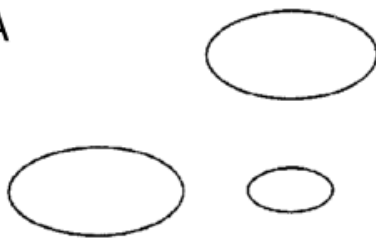
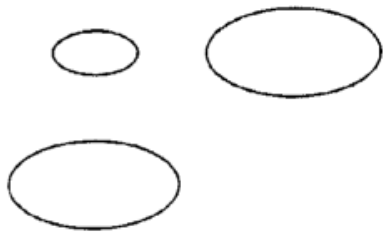

B

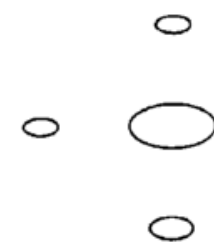

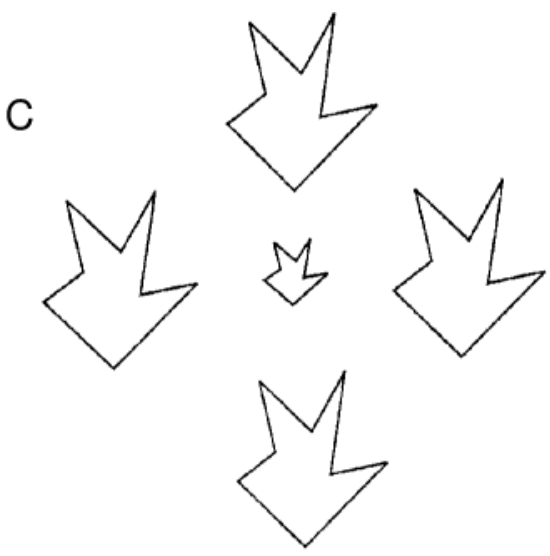

M

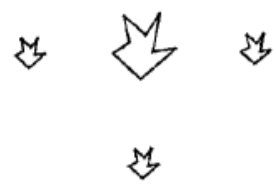

Figure 1. Two examples of sets of stimuli. The central stimuli of each set are identical in size. The surrounding stimuli differ in size, however, thus creating the Ebbinghaus illusion.

target looks smaller than when the context is composed of relatively small stimuli (see, e.g., Choplin \& Medin, 1999; Coren \& Enns, 1993; Coren \& Miller, 1974; Stapel \& Koomen, 1997.)

According to the present hypothesis, the perceived features of objects that take part in a similarity-oriented comparison are more similar than the perceived features of (the same) objects that take part in a difference-oriented comparison. It is hypothesized, then, that the Ebbinghaus illusion following similarity-oriented comparisons should be weaker than the Ebbinghaus illusion following difference-oriented comparisons. The "regular" Ebbinghaus illusion - that is, the one obtained when one does not engage in comparison processes prior to estimating the size of the target objects - should fall somewhere in between.

\section{METHOD}

\section{Participants}

Ninety-five students from Tel Aviv University (37 males, 58 females) participated in the study in partial fulfillment of a course requirement. Their mean age was 23 .

\section{Materials}

Eighteen pairs of stimuli were created, each containing a target and a context of inducing objects (see the examples in Figure 1). In one stimulus of each pair, the inducing objects were bigger than the target object, whereas in the other they were smaller than the target. A pretest examined the magnitude of the Ebbinghaus illusion (MEI) that these pairs create, and the 10 pairs that created the biggest illusion were chosen for the study itself.

\section{Procedure}

The participants took part in the study either individually or in small groups. They were randomly assigned to one of the six con- ditions created by two independent variables: context size (small vs. big context objects) and type of comparison (similarity, difference, and control). Each participant received a booklet containing 10 stimuli, each appearing on a separate page. They were told that they were taking part in a study that would examine "visual processing and cognitive judgments." At the bottom of each page appeared a horizontal line that had a short vertical stop at the far left end. The participants were asked to indicate the widest horizontal extent of each target object. This was done by placing a pen mark so that the distance between the left end of the line and the mark was equal to the perceived horizontal extent of the target object. Prior to estimating the size, the participants in the similarity and difference conditions were asked to judge the similarity (difference) of the target and the context objects, using a 9-point scale ranging from very similar (very different) to not similar at all (not different at all). The participants in the control condition were asked to judge how complex the objects were before engaging in the estimation of size.

\section{Scoring}

A "blind" judge used a ruler to measure (in millimeters) the length of the horizontal lines marked by the participants.

\section{RESULTS}

The MEI is measured by subtracting the mean perceived size of targets in the big-context condition from their mean perceived size in the small-context condition. In the control condition, when no explicit comparison took place prior to the estimation of size, the MEI was $2.10 \mathrm{~mm}$ (see Table 1). The Ebbinghaus illusion virtually disappeared in the similarity condition $(\mathrm{MEI}=0.10 \mathrm{~mm}$ ) and grew slightly larger in the difference condition (MEI = $2.56 \mathrm{~mm})$.

A 2 (context, big vs. small) $\times 3$ (type of comparison, similarity vs. difference vs. control) analysis of variance, 
Table 1

Mean Sizes (in Millimeters) of Focal Stimuli as a Function of Comparison Orientation and the Size of the Contextual Figures

\begin{tabular}{lccccc}
\hline & \multicolumn{2}{c}{ Small Context } & & \multicolumn{2}{c}{ Big Context } \\
\cline { 2 - 3 } \cline { 5 - 6 } Condition & $M$ & $S D$ & & $M$ & $S D$ \\
\hline Similarity & 20.01 & 2.35 & & 19.91 & 1.86 \\
Difference & 21.04 & 2.42 & & 18.48 & 2.01 \\
Control & 20.93 & 1.85 & & 18.83 & 2.65 \\
\hline
\end{tabular}

conducted on the mean sizes of the target objects, showed a significant interaction, $[F(1,90)=4.18, p<.05]$. In order to directly examine the hypothesis, a planned comparison between the MEIs in the difference and the similarity conditions was conducted, revealing a significant effect $[F(1,90)=7.00, p<.01]$. Further comparisons revealed that the MEIs in the similarity and control conditions were significantly different $[F(1,90)=5.43, p<$ $.05]$, whereas the MEIs in the difference and the control conditions did not significantly differ $[F(1,90)=0.1, p>$ .75].

Since the MEI in the similarity condition significantly differed from that in the control condition, whereas that in the difference condition did not, it seems that most of the action occurred in the similarity-oriented comparisons. This tentative conclusion might be premature, however. One alternative explanation to be considered is that the action in the difference-oriented condition was weaker simply because the stimuli were relatively similar to each other. This explanation is supported by the actual ratings of similarity and difference. The mean rating of similarity was $7.48(S D=0.282)$, whereas the mean rating of difference was $4.14(S D=0.245)$.

\section{DISCUSSION}

Both philosophers (e.g., Goodman, 1982) and psychologists (e.g., Murphy \& Medin, 1985; Shanon, 1988) have attacked the notion of similarity in general and feature-based similarity in particular, claiming that it is too unconstrained and, hence, cannot perform sufficient explanatory work. Accordingly, noting that every pair of objects (or concepts, people, etc.) share any number of features and also differ in any number of features, philosopher Nelson Goodman claims that similarity is an illdefined notion that has no meaning unless relevant respects of the comparison are specified. But, he goes on, if similarity has no meaning in itself and all the work is done by determination of the respects in which the comparison is to be made, it becomes a vacuous concept (Goodman, 1982). Hence, either similarity is "a pretender, an impostor, a quack" (p. 437), or else one has to offer ways in which similarity comparisons can be made meaningful. One of the ways to make similarity more meaningful is to treat it as a constructive process, one that not only takes into account objects and features, but also constrains features and creates respects for the comparison.
The present study provides new evidence for the constructive nature of comparison processes (see also Markman \& Gentner, 1993, 1996; Medin et al., 1993). First, it demonstrates that comparison processes affect perception, as measured by participants' width estimates. Second, it demonstrates that different orientations of comparison processes affect perception differently. Relative to the difference orientation (and the control), similarity orientation makes the features of the objects in the comparison more similar to each other. The present findings suggest, then, that in order to understand comparison processes, it is not enough to ask how features (attributes, relations) construct similarity. One must also ask how comparison processes construct features.

As was noted in the introduction, similarity and difference judgments are not always inverses. Three mechanisms of differential weighting have been proposed to explain findings of this sort. Tversky (1977) suggested that people may attend more to common features in similarity judgments, whereas they may attend more to distinctive features in difference judgments. Medin et al. (1990) suggested that similarity judgments emphasize relations, whereas difference judgments emphasize attributes. Lastly, Markman (1996) suggested that shared relations are more important to similarity judgments than are shared attributes, whereas alignable differences are more important to difference judgments than are nonalignable differences (e.g., Markman \& Gentner, 1996). The perceptual effects of comparison processes, however, may suggest a different kind of mechanism for noncomplementary similarity and difference judgments. According to this suggestion, similarity and difference judgments are sometimes not complementary simply because similarity judgments enhance perceptual similarity, whereas difference judgments enhance perceptual difference.

In previous investigations of the Ebbinghaus illusion, the role of similarity between the target and the inducing objects was examined. Coren and his colleagues (Coren \& Enns, 1993; Coren \& Miller, 1974) provided evidence in support of the claim that the Ebbinghaus illusion is a function of the similarity between the inducing objects and the target. These results were replicated and extended by Stapel and Koomen (1997), who showed that the MEI is sensitive to similarities in the social categorizations of the stimuli. Recent research by Choplin and Medin (1999), however, suggests a dissociation between the MEI and similarity. Can the present findings shed new light on the intricate relationships between comparison processes and the Ebbinghaus illusion? The findings reported above show that a change in the orientation of the comparison process results in changes in MEIs. This finding, then, seems to support the former view, according to which the Ebbinghaus illusion is correlated with similarity. However, because the stimuli used in the study were geometrical shapes, in which the perimeter is salient, and because even Choplin and Medin think that perimeter similarity might be correlated with the Ebbinghaus illusion, the exact nature of the relations 
between similarity and the Ebbinghuas illusion is left for future investigations.

\section{REFERENCES}

Andersen, S. M., Glassman, N. S., Chen, S., \& Cole, S. W. (1995). Transference in social perception: The role of chronic accessibility in significant-other representations. Journal of Personality \& Social Psychology, 69, 41-57.

Choplin, J. M., \& Medin, D. L. (1999). Similarity of the perimeters in the Ebbinghaus illusion. Perception \& Psychophysics, 61, 3-12.

Coren, S., \& ENNS, J. T. (1993). Size contrast as a function of conceptual similarity between test and inducers. Perception \& Psychophysics, 54, 579-588.

Coren, S., \& Miller, J. (1974). Size contrast as a function of figural similarity. Perception \& Psychophysics, 16, 355-357.

Gentner D., \& Markman A. B. (1994). Structural alignment in comparison: No difference without similarity. Psychological Science, $\mathbf{5}$, $152-158$.

Goodman, N. (1982). Seven strictures on similarity. In N. Goodman (Ed.), Problems and projects (pp. 437-447). New York: Bobbs-Merrill.

HolyOAK, K. J., \& KOH, K. (1987). Surface and structural similarity in analogical transfer. Memory \& Cognition, 15, 332-340.

MARKMAN, A. B. (1996). Structural alignment in similarity and difference judgments. Psychonomic Bulletin \& Review, 3, 227-230.

Markman, A.B., \& Gentner, D. (1993). Splitting the difference: A structural alignment view of similarity. Journal of Memory \& Language, 34, 517-535.

Markman, A. B., \& Gentner, D. (1996). Commonalities and differences in similarity comparisons. Memory \& Cognition, 24, 235-249.

Medin, D. L., Goldstone, R. L., \& Gentner D. (1990). Similarity involving attributes and relations: Judgments of similarity and difference are not inverses. Psychological Science, 1, 64-69.
Medin, D. L., Goldstone, R. L., \& Gentner, D. (1993). Respects for similarity. Psychological Review, 100, 254-278.

Murphy, G. L., \& Medin, D. L. (1985). The role of theories in conceptual coherence. Psychological Review, 92, 289-316.

Ortony, A. (1979). Beyond literal similarity. Psychological Review, 86, $161-180$.

OsGood, C. E. (1949). The similarity paradox in human learning: A resolution. Psychological Review, 56, 132-143.

Osherson, D. N., Smith E. E., Wilkie, O., Lopez, A., \& Shafir, E. (1990). Category based induction. Psychological Review, 97, 185-200.

Shanon, B. (1988). On the similarity of features. New Ideas in Psychology, 6, 307-321.

Smith, E. E., \& Medin, D. L. (1981). Categories and concepts. Cambridge, MA: Harvard University Press.

Stapel D. A., \& Koomen, W. (1997). Social categorization and perceptual judgment of size: When perception is social. Journal of Personality \& Social Psychology, 73, 1177-1190.

Tversky, A. (1977). Features of similarity. Psychological Review, 84, 327-351.

\section{NOTE}

1. Think of the following examples. One cannot notice that "Julia Roberts and my cocker spaniel have similar eyes" without realizing some differences (e.g., the eyes are placed on different bodies) because if it was not for these differences, there would have been only one pair of eyes. Nor can one observe that "Julia's smile is much more heartwarming than my cocker spaniel's" without noticing some similarities (e.g., both involve moving the corners of the mouth upwards), for if it was not for these similarities, one would not have known that both are smiles.

(Manuscript received November 17, 1999; revision accepted for publication September 5, 2000.) 